\title{
The art of medicine in treating osteoarthritis: I will please
}

\section{J W J Bijlsma, P M J Welsing}

The Latin word placebo literally means, "I will please". Until 1945 the placebo was a "morally" useful but innocuous management tool without curative or symptomatic consequences. When in the 1950s the double-blind randomised controlled trial (RCT) began to establish itself, the placebo was imbued with powerful therapeutic effects and its ethical use in clinical practice was being questioned. ${ }^{1}$ In a few years the placebo changed from what was called the "humble humbug" (a means of reinforcing a patient's confidence in his recovery) to an entity with occult-like powers that could mimic potent drugs.

Nowadays placebo is primarily used within the RCT setting; use in clinical practice is generally considered unethical. In the current literature placebo is described as "inert", "inactive" or "nonspecific" and as "dummy" or "sham" treatment in the context of a RCT. An "active" treatment not expected to be useful for the complaints at hand, however, might work on the same principal. When we ask experienced doctors for their opinion on observed placebo effect we receive quite some contradictory responses. Conflicting explanations such as expectation, anxiety relief, faith, patient-doctor relationship, self-perceptions, classic conditioning, symbolic processes, holistic approach, fraud and tricking patients are mentioned among others.

\section{PLACEBO EFFECTS IN OSTEOARTHRITIS}

Some of these feelings became apparent in the recent discussion of treatment of osteoarthritis (OA) with glucosamin. A long awaited "definite" trial, the GAIT (Glucosamin/chondroitin Arthritis Intervention Trial) had some remarkable results. ${ }^{2}$ In this study, patients with symptomatic (with more then half also radiological) knee OA were randomised to five treatment groups: glucosamin, chondroitin sulphate, a combination of

Department of Rheumatology \& Clinical Immunology, University Medical Center Utrecht, Utrecht,

The Netherlands

Correspondence to: Professor J W J Bijlsma, University Medical Center Utrecht, The Netherlands; J.W.J.Bijlsma@umcutrecht.nl glucosamin and chondroitin sulphate, celecoxib and placebo. Primary outcome was a decrease of at least $20 \%$ in pain measured by the Western Ontario and McMaster Universities (WOMAC) score. This outcome was reached in $64 \%$ of the glucosamin group, $65 \%$ of the chondroitin sulphate group, $67 \%$ of the combination, $70 \%$ of the celecoxib and $60 \%$ of the placebo group.

Perhaps triggered by the ensuing discussion about the placebo effect, Zhang et al set themselves the task of systematically reviewing the literature on placebo response in the treatment of OA. ${ }^{3}$ Their interesting report is published in this issue of the Annals of Rheumatic Diseases (see page 1716). If their conclusion is valid, then the reported high placebo response in the GAIT seems not to be an exception, but rather the rule.

For their analysis Zhang et al defined the placebo effect as the change from baseline to endpoint in the placebo group, estimated as the effect size (ES; ie, the standard mean difference). The primary outcome was the ES for pain. Clinically, an ES of 0.20 suggests a small effect, 0.50 a moderate effect and over 0.80 indicates a large effect. The systematic literature search revealed 198 trials, of which 14 included untreated controls. The ES as found in the placebo group was compared with the ES obtained from untreated controls. The ES in the untreated group was 0.03 . In the placebo arms of the trials the ES was 0.51. Zhang et al conclude: "Placebo is effective in the treatment of OA, especially for pain, stiffness and self-reported function". ${ }^{3}$

Notwithstanding difficulties in precisely estimating the size of the placebo effect, the differences between placebo groups and non-treatment groups as found by Zhang et al are much larger then those between placebo and active treatment groups; though there is heterogeneity between different studies, outcomes and treatment modalities. The reported mean ES for pain of placebo (0.51) across the trials is impressive, as are the ES for function (0.49), stiffness (0.43) and doctor global score (ES 0.66). Placebo becomes difficult to beat in randomised placebo controlled trials in osteoarthritis!
There were only a limited number of studies in their analysis with more objective endpoints, such as walking time, joint space width and others. The observed ES of placebo becomes smaller in those trials. Another interesting finding is the difference between placebo and active treatment groups based on the site of OA: the ES of placebo is twice as high in hand $O A$ (0.80) compared to hip OA (ES $=0.37)$.

\section{PLACEBO EFFECTS IN OTHER STUDIES}

In an analysis of clinical trials in different diseases, comparing placebo with no treatment, there was only little evidence reported that placebos may have powerful clinical effects. ${ }^{4}$ In all, 32 studies reported binary outcomes in 3795 patients, and 82 studies reported continuous outcomes involving 4730 patients. As compared with no treatment, placebo had no significant effect on binary outcomes, regardless of whether these outcomes were subjective or objective. For the trials with continuous outcomes, placebo had a beneficial effect. The effect decreased with increasing sample size, indicating a possible bias related to the effects of small trials. In this metaanalysis the effect of placebo in trials with continuous outcomes was expressed as the standardised mean difference, with a beneficial effect for placebo on subjective outcomes (among which pain) of 0.36 and of objective outcomes of 0.12 . The effects of placebo were greater in the psychological (0.34) and physical (0.31) then in the pharmacological (0.20) treatments. A reanalysis ${ }^{5}$ of the clinical trials comparing placebo with no treatment ${ }^{4}$ concluded that in conditions amendable for the placebo effect and studies using an adequate design, the placebo effect was robust and approaches the treatment effect. Which factors may influence these findings and conflicting conclusions? Studying the placebo effect constitutes several challenges of which some relate to the definition of the placebo effect and others to the "science of effect estimation".

\section{DEFINITION OF THE PLACEBO EFFECT}

The placebo effect can be defined in many ways: as a causal effect or as just the temporal effect after receiving placebo, or as the effect of the patient-provider interaction and/or of specific component(s) of this interaction. ${ }^{6}$ We can define the placebo effect over all patients, diseases, outcomes and treatments or limit it within a more specific context as Zhang et al did. Some of the contradictory conclusions as described 
above can be explained by considering these different definitions.

When we want to estimate the "real" placebo effect and distinguish it from effects such as regression to the mean and the natural course of the diseases we need a "no treatment" control group. Ideally a direct comparison within a single RCT would be used. Usually there are not many of these studies within a specific disease area. Only three of these studies were found in the analysis of Zhang et al but they did confirm the result of the total study.

Comparing the placebo response with the response in the non-treatment groups is not sufficient to rule out all bias or to differentiate between different components of the placebo effect. Of course a patient may feel disappointed by clearly not being treated. This might constitute a negative placebo effect (which is actually part of the placebo effect) also known as the nocebo effect ("I will harm"). Whether placebo is a realistic option is more or less dictated by the "active" treatment in the trial; it is more difficult to think about a good placebo for exercises then for drugs. Though trial blinding in studies comparing placebo with hyaluronic acid on the one hand and glucocorticoids on the other are probably comparable, the ES of placebo in the first group was 0.73 and in the glucocorticoid group 0.39. Possibly, systemic effects of glucocorticoids experienced by the patients in the last group might partially explain this difference. When the placebo effect is defined as the patient-provider interaction, a non-treatment group is hard to define; there will always be some interaction. Further factors such as reporting bias (a patient might feel obliged to report an effect when there really is not any, again: "I will please"), publication bias and concomitant treatment might play a role as well.

So the placebo effect is real, although it might not be equally large or even present in all situations. In other words the "real" placebo effect is context specific. Studying this so-called "self-healing effect" or "contextual healing" apart from scientific effect estimation of the placebo effect may yield useful insights for clinical practice.

\section{PATIENT-DOCTOR RELATIONSHIP}

An important contribution in understanding the different components of placebo effect was recently provided from a RCT in patients with irritable bowel syndrome. $^{7}$ In 262 adults the effects of placebo acupuncture were evaluated in circumstances that involved observation only, sham acupuncture alone and an enriched relationship with the doctor, along with the sham procedure. The proportion of patients that reported moderate or substantial improvement of the irritable bowel syndrome on global improvement scale was 3\% in the observation group, $20 \%$ in the procedure alone group and $37 \%$ in the augmented intervention group. Clearly the group with the greatest relief of symptoms was the one that received not only sham acupuncture but also 45 min of quality contact with a clinician. It was concluded that the doctor-patient relationship is a crucial part of the placebo effect.

\section{OTHER ASPECTS}

Another recent study highlighted the commercial features of placebo and therapeutic efficacy. In a randomised controlled setting the effect of price on analgesic response to placebo pills was evaluated. ${ }^{8}$ A total of 82 healthy paid volunteers were informed about a purported new opioid analgesic, similar to codeine, but with a faster onset time; it was in fact a placebo. Half of the participants were told that the drug had a regular price of US $\$ 2.50$ per pill, and the other half a discounted price of US $\$ 0.10$. Electrical shocks were given to the participants, and they rated their pain by voltage intensity. Considering all voltages tested, pain reduction was greater for the expensive pill then for the discounted pill. Pain relief by this placebo was $61 \%$ for the cheap pill and $85 \%$ for the expensive pill.

Time trends may also exist with the placebo. As found in studies on major depression placebo responses have increased over time, and (to a lesser extent) also in the active treatment arms. ${ }^{9}$ This was attributed by the authors to milder, more responsive patients included in studies nowadays. The placebo response may also increase by increasing expectations of newer healthcare interventions. By contrast, in tobacco addiction placebos, the response declined over time, which was attributed to more recalcitrance of smokers in clinical trials over time. ${ }^{10}$

In addition, the purpose of a study might influence the size of the placebo effect. Studies in which the purpose was to study the placebo effect itself found higher effect sizes than studies in which placebo was only used as control. It was also reported that when a placebo analgesic effect is induced via suggestion and conditioning the magnitude of the placebo response was higher than via suggestion or conditioning alone. ${ }^{11}$

Importantly, the placebo effect can be evoked without the use of an actual placebo. This was elegantly shown by comparing open and hidden administration of different analgesic drugs. The therapeutic power of various analgesic drugs was markedly reduced when administered by a computer controlled infusion pump without the patient knowing that the drug is being given, as compared with open administration of the drug by a clinician, described to the patient as a pain relieving intervention. ${ }^{12}$

Recent brain imaging research on pain and the placebo effect has elucidated a physiological reflection of the placebo effect by showing the functional connectivity between specific brain regions that process attention (the anterior cingulated gyrus) and pain (periaqueductal grey) involving endogenous opiate receptors. ${ }^{13}$

\section{IN SUMMARY}

Many factors, some expected and others unexpected, play an important role in the placebo effect. Placebo responses on pain are among the most studied, and usually found to be considerable. The placebo effect is something we not only have to live with and control for in clinical studies, but something that we can optimise in the care of our patients. Some of these insights might long be used implicitly or explicitly and conscious or unconscious by complementary medicine practitioners and in this respect we might learn from them.

How to incorporate the findings of Zhang et al in our daily clinical practice? First, we should realise that placebo effect is not a negative effect. We should realise that the effect of a "real" intervention is the result of the "active" treatment plus the placebo effect, although it has been challenged whether the effects are in fact additive. ${ }^{14}$ We should make proper use of that finding. Clearly, the doctor-patient relationship is a very powerful tool in the art of medicine, and is probably a cost effective part of the placebo effect. Primum nil nocere (in the first place, do not do any harm) is also a powerful adagium of the art of medicine; making optimal use of the placebo effect is in line with this statement. So our conclusion would be: there is no need to use actual placebos, but there is nothing wrong in using the placebo effect in treating patients. Patients with OA, in whom pain plays a crucial role, will benefit from doctors who are able to use the placebo effect in a respectable way, "to please the patient".

\section{Competing interests: None declared.}

Accepted 18 September 2008

Ann Rheum Dis 2008;67:1653-1655.

doi:10.1136/ard.2008.097006 


\section{REFERENCES}

1. Kaptchuk TJ. Powerful placebo: the dark side of the randomized controlled trial. Lancet

1998;351:1722-25.

2. Clegg DO, Reda DJ, Harris CL, Klein MA, O'Dell JR, Hooper MM, et al. Glucosamine, chondroitin sulfate, and the two in combination for painful knee osteoarthritis. N Engl J Med

2006:354:795-808.

3. Zhang W, Robertson J, Jones AC, Dieppe PA, Doherty $M$. The placebo effect and its determinants in osteoarthritis: meta-analysis of randomised controlled trials. Ann Rheum Dis 2008;67:1716-23.

4. HrobjartssonA, Gotze PC. Is the placebo powerless? An analysis of clinical trials comparing placebo with no treatment. $N$ Engl J Med 2001:344:1594-602.
5. Wampold BE, Minami T, Tierney SC, Baskin TW, Bhati KS. The placebo effect is powerful: estimating placebo effects in medicine and psychotherapy randomized clinical trials. J Clin Psychology 2005;61:835-54.

6. Hrobjartsson A. What are the main methodological problems in the estimation of placebo effects? J Clin Epidemiology 2002;55:430-5.

7. Kaptchuk TJ, Kelley JM, Conboy LA, Davis RB, Kerr $\mathrm{CE}$, Jacobson EE, et al. Components of placebo effect: randomized controlled trial in patients with irritable bowel syndrome. BMJ. Published Online First: 3 April 2008. doi:10.1136/bmj.39524. 439618.25

8. Waber RL, Shiv B, Carmon Z, Ariely D. Commercial features of placebo and therapeutic efficacy. JAMA 2008;299:1016-7.
9. Walsh BT, Seidmann SN, Sysko R, Gould M. Placebo response in studies of major depression. Variable, substantial, and growing. JAMA 2002;287:1840-7.

10. Irvin JE, Hendricks PS, Brandon TH. The increasing recalcitrance of smokers in clinical trials II: pharmacotherapy trials. Nicotine Tob Res 2003:5:27-35.

11. Vase L, Riley JL, Price DD. A comparison of placebo effects in clinical analgesic trials versu studies of placebo analgesia. Pain 2002;99:443-52.

12. Colloca L, Lopiano L, Lanotte M, Benedetti F. Overt versus covert treatment for pain, anxiety and Parkinson's disease. Lancet Neurol 2004;3:679-84.

13. Wager TD, Scott DJ, Zubieta JK. Placebo effects on human mu-opioid activity during pain. Proc Natl Acad Sci USA 2007;104:11056-61.

14. Spiegel D, Harrington $A$. What is the placebo effect worth? BMJ 2008:336:967-8. 
patients suffered severe bacterial or opportunistic infections; serum immunoglobulin levels remained within the normal limits during treatment in all cases.

Our preliminary results suggest that rituximab seems to be a useful therapeutic alternative in patients with active SOJIA in whom previous treatments (including TNF $\alpha$ antagonists and anakinra) have failed. Our experience is that rituximab produces a substantial clinical improvement (remission of the systemic symptoms and moderate European League Against Rheumatism response of the arthritis), although the disease does not enter into remission. Of interest, our experience with rituximab is similar to the French experience with IL-1 receptor antagonist treatment in SOJIA/adult-onset Still's disease. ${ }^{6}$ In that study, the benefits with anti-IL-1 therapy seem to be fair in the systemic manifestations but reduced in the articular complaints.

Further studies are needed to determine the place of specific B-cell depletion in the treatment of refractory SOJIA.

\section{J Narváez, ${ }^{1}$ C Díaz-Torné, ${ }^{2}$ X Juanola, ${ }^{1}$ C Geli, ${ }^{2} \mathrm{~J} \mathrm{M} \mathrm{Llobet,}{ }^{2}$ J M Nolla, ${ }^{1}$ C Díaz-López ${ }^{2}$}

${ }^{1}$ Department of Rheumatology, Hospital Universitario de Bellvitge-IDIBELL, Barcelona, Spain; ${ }^{2}$ Department of Rheumatology, Hospital de la Santa Creu i Sant Pau, Barcelona, Spain
Correspondence to: Dr F J Narváez García, Department of Rheumatology (planta 10-2), Hospital Universitario de Bellvitge, Feixa Llarga s/n 08907, L'Hospitalet de Llobregat, Barcelona, Spain; 31577edd@comb.es

Competing interests: None.

Ethics approval: Ethics approval was obtained.

Patient consent: Obtained.

Accepted 26 July 2008

Ann Rheum Dis 2009;68:607-608. doi:10.1136/ard.2008.092106

\section{REFERENCES}

1. Adams A, Lehman TJ. Update on the pathogenesis and treatment of systemic onset juvenile rheumatoid arthritis. Curr Opin Rheumatol 2005;17:612-16.

2. Ramanan AV, Grom AA. Does systemic-onset juvenile idiopathic arthritis belong under juvenile idiopathic arthritis?. Rheumatology 2005;44:1350-3.

3. Ohlsson V, Baildam E, Foster H, Jandial S, Pain C, Strike H, et al. Anakinra treatment for systemic onset juvenile idiopathic arthritis (SOJIA). Rheumatology 2008;47:555-6.

4. Eberhard BA, llowite NT. Response of systemic onset juvenile rheumatoid arthritis to etanercept: is the glass half full or hald empty?. J Rheumatol 2005;32:763-5.

5. Billiau AD, Cornillie F, Wouters C. Infliximab for systemic onset juvenile idiopathic arthritis: experience in 3 children. J Rheumatol 2002:29:1111-14.

6. Lequerré T, Quartier P, Rosellini D, Alaoui F, De Bandt M, Mejjad O, et al. Interleukin-1 receptor antagonist (anakinra) treatment in patients with systemic-onset juvenile idiopathic arthritis or adult onset Still disease: preliminary experience in France. Ann Rheum Dis 2008;67:302-8.

7. Ahmadi-Simab K, Lamprecht $P$, Jankowiak C, Gross WL. Successful treatment of refractory adult onset Still's disease with rituximab. Ann Rheum Dis 2006;65:1117-18.

\section{Corrections}

The cover caption for the supplement published in December 2008 (volume 67, suppl iii) was inadvertently missed from the contents page. It should have read "Part of "Augustine Roulin with her infant' by Vincent van Gogh (1889). Mrs Roulin suffered from psoriatic arthropathy. Metropolitan Museum of Art, New York, USA."

The authors JWJ Bijlsma and PMJ Welsing of the editorial "The art of medicine in treating osteoarthritis: I will please" (Ann Rheum Dis 2008;67:1653-5) regret that a reference to an important work by Dr Franklin G Miller (Department of Bioethics, National Institute of Health) and co-workers was not included on p1654, first paragraph, right column. This part explains findings reported in reference 12 and also text extracted from the following reference: Miller FG, Kaptchuk TJ. The power of context: reconceptualizing the placebo effect. $J R$ Soc Med 2008;101:222-5. This reference should have been included together with reference 12 . 